\title{
BMJ Open Evaluation of the McGrath MAC and Macintosh laryngoscope for tracheal intubation in 2000 patients undergoing general anaesthesia: the randomised multicentre EMMA trial study protocol
}

\author{
Marc Kriege, ${ }^{1}$ Christian Alflen, ${ }^{1}$ Irene Tzanova,${ }^{2}$ Irene Schmidtmann, ${ }^{3}$ Tim Piepho, ${ }^{1}$ \\ Ruediger R Noppens ${ }^{1,4}$
}

To cite: Kriege M, Alflen C, Tzanova I, et al. Evaluation of the McGrath MAC and Macintosh laryngoscope for tracheal intubation in 2000 patients undergoing general anaesthesia: the randomised multicentre EMMA trial study protocol. BMJ Open 2017;7:e016907. doi:10.1136/ bmjopen-2017-016907

- Prepublication history for this paper is available online. To view these files please visit the journal online (http://dx.doi. org/10.1136/bmjopen-2017016907).

Received 17 March 2017 Revised 29 May 2017 Accepted 6 June 2017

CrossMark

${ }^{1}$ Department of Anaesthesiology, University Medical Centre of the Johannes, Gutenberg University, Mainz, Germany

${ }^{2}$ Department of Anaesthesiology, Christophorus Hospital,

Coesfeld, Germany

${ }^{3}$ Institute of Medical

Biostatistics, Epidemiology

and Informatics, University

Medical Centre of the Johannes

Gutenberg University, Mainz,

Germany

${ }^{4}$ Department of Anesthesia and Perioperative Medicine, Western University, London, Ontario, Canada

Correspondence to Dr Ruediger R Noppens; ruediger.noppens@|hsc.on.ca

\section{ABSTRACT}

Introduction The direct laryngoscopy technique using a Macintosh blade is the first choice globally for most anaesthetists. In case of an unanticipated difficult airway, the complication rate increases with the number of intubation attempts. Recently, McGrath MAC (McGrath) video laryngoscopy has become a widely accepted method for securing an airway by tracheal intubation because it allows the visualisation of the glottis without a direct line of sight. Several studies and case reports have highlighted the benefit of the video laryngoscope in the visualisation of the glottis and found it to be superior in difficult intubation situations. The aim of this study was to compare the first-pass intubation success rate using the (McGrath) video laryngoscope compared with conventional direct laryngoscopy in surgical patients.

Methods and analysis The EMMA trial is a multicentre, open-label, patient-blinded, randomised controlled trial. Consecutive patients requiring tracheal intubation are randomly allocated to either the McGrath video laryngoscope or direct laryngoscopy using the Macintosh laryngoscope. The expected rate of successful first-pass intubation is $95 \%$ in the McGrath group and $90 \%$ in the Macintosh group. Each group must include a total of 1000 patients to achieve $96 \%$ power for detecting a difference at the $5 \%$ significance level. Successful intubation with the first attempt is the primary endpoint. The secondary endpoints are the time to intubation, attempts for successful intubation, the necessity of alternatives, visualisation of the glottis using the Cormack \& Lehane score and percentage of glottic opening score and definite complications.

Ethics and dissemination The project was approved by the local ethics committee of the Medical Association of the Rhineland Palatine state and Westphalia-Lippe. The results of this study will be made available in the form of manuscripts for publication and presentations at national and international meetings.

Trial registration number ClinicalTrials.gov NCT 02611986; pre-results.
Strengths and limitations of this study

- This trial aims to determine whether video laryngoscopy is superior to direct laryngoscopy in daily anaesthesia practice.

- The plan is to include 2000 patients in this multicentre, open-label, randomised controlled superiority study.

- All training levels of anaesthesiologists (trainee, specialist, expert) are included.

- Selected patients with an expected normal airway are evaluated.

- One type of video laryngoscope using a Macintoshlike blade is evaluated. The results cannot be transferred to other kinds of video laryngoscopes using different kind of blades (eg, hyperangulated blade, channelled blade).

\section{INTRODUCTION}

\section{Background and rationale}

Securing the airway by tracheal intubation with direct laryngoscopy (DL) is an established and preferred technique in emergency settings and clinical anaesthesia practice. The limitations of DL are well known. To achieve a learning curve with a $90 \%$ probability of performing a successful intubation, more than 57 attempts are required to develop enough experience with the technique. ${ }^{12}$ To obtain optimal visualisation of the glottis, DL requires alignment of the oropharyngeal-laryngeal axes.

However, the first-pass success rate of intubation in emergency settings ranges from $40 \%$ to $80 \%,{ }^{3-7}$ in intensive care units from $55 \%$ to $68 \%^{8-10}$ and in the operating room from $63 \%$ to $85 \% .{ }^{11-14}$ Several studies have shown a correlation between increased complications and more than two intubation attempts. ${ }^{15-17}$ 
Indirect video laryngoscopy has become a widely accepted method for learning the techniques of airway management because it enables an optimised view of the glottis without a direct line of sight. ${ }^{18-20}$ Thus, video laryngoscopy plays an important role in the management of patients with unanticipated airway difficulties or failed tracheal intubation. The use of video laryngoscopy is associated with a reduction in airway complications in clinical emergency and anaesthesia practice. ${ }^{17}{ }^{21}$ Despite the optimised visualisation of the glottis, the duration of tracheal intubation can be prolonged and intubation attempts can fail. ${ }^{20-23}$ Compared with DL, the learning curve associated with the video laryngoscope is steep. ${ }^{24}$ Video laryngoscopy varies in the design of the curved or angulated blade, mobility, size of the monitor display and operation of the micro camera on the tip of the blade.

Over the last decade, several studies have compared different video laryngoscopes with DL or against each other, focusing on endotracheal intubation (ET) in emergency settings or in patients undergoing elective surgery in an operating room. The results suggested advantages in superior visualisation of the glottis, ${ }^{18} 2022$ a higher first-pass success rate $^{3101823}$ and reduction of airway complications, as well as benefits in those patients with a difficult airway. ${ }^{20-22}$ However, most of these studies had methodological weaknesses, including studies with small sample sizes, ${ }^{5}{ }^{12}$ 18-20 23 evaluation in intensive care units $^{369102526}$ or emergency departments, ${ }^{457}$ manikin studies, ${ }^{19} 20$ and inclusion of patients who were anticipated to have a difficult airway. ${ }^{11} 18-22$

Studies conducted more recently have suggested advantages using video laryngoscopy but either failed to routinely use neuromuscular blockade, ${ }^{3}$ i or included patients with highly specific characteristics. ${ }^{4}$ Special study characteristics are listed in table 1 .

We chose to study the McGrath MAC (McGrath; Medtronic, Dublin, Ireland) video laryngoscope because it is a portable, relatively inexpensive device with a Macintosh-based blade similar to that in the Macintosh laryngoscope (DL; Stoss Medica, Wiesbaden, Germany). It therefore provides both: a direct view of the glottis and an indirect view on the monitor display, which can be beneficial in the case of oropharyngeal mismatch. Our specific choice of the McGrath video laryngoscope was based on the following considerations:

- The Macintosh-based curved blade of the McGrath is comparable to the Macintosh blade.

- The video display of the McGrath allows visualisation of the glottis by the operator along with study measurement or teaching by a consultant when tracheal intubation is performed by an inexperienced provider.

- The McGrath is available with a disposable blade in different sizes and allows a swift change to treat more patients consecutively.

The aim of this study is to evaluate whether the use of the McGrath improves the first-pass success rate compared with DL in surgical patients with an expected normal airway undergoing general anaesthesia. We hypothesise that tracheal intubation using the McGrath decreases the frequency of failed intubation and airway complications.

\section{Study aims and objectives \\ Primary objective}

The primary objective is to compare the initial or first-pass success rate of ET with the McGrath video laryngoscope with DL using a Macintosh blade in patients undergoing elective surgery and requiring tracheal intubation.

\section{Secondary objective}

The secondary objective is to compare the clinical performance of both devices, view of the glottis, influence of neuromuscular agents, correlation between clinical experiences in airway management and success rates.

\section{Trial design}

The EMMA trial is a multicentre, open-label, randomised controlled superiority trial.

\section{METHODS}

Participant selection, interventions and outcomes

This manuscript was written in accordance with the SPIRIT guidelines. ${ }^{27}$

\section{Study setting}

The EMMA trial is performed in eight divisions of anaesthesiology in two hospitals (one university and one general hospital). All laryngoscopists are anaesthetists with different levels of clinical experience using direct and video laryngoscopy. After a specific introduction to the study protocol, all anaesthetists from the study centres participate in this trial.

\section{Eligibility criteria}

\section{Inclusion criteria}

Patients having elective surgery with general anaesthesia and requiring mechanical ventilation via an endotracheal tube are recruited.

\section{Exclusion criteria}

Patients are not included in this study if they meet one or more of the following criteria:

- More than one predictor of an anticipated difficult airway (eg, body mass index (BMI) $>40 \mathrm{~kg} / \mathrm{m}^{2}$, unanticipated difficult airway in the medical history (eg, Cormack \& Lehane $(\mathrm{C} \& \mathrm{~L}) \geq \mathrm{III})$, reduction of the atlanto-occipital joint extension $<35^{\circ}$, reduced thyromental distance $<6 \mathrm{~cm}$ or Mallampati class $\geq \mathrm{III}$ )

- Age $<18$ years

- ASA class IV

- Pregnant or breast feeding

- Participation in other studies

- Unable to provide informed written consent or under guardianship

- Urgent surgical intervention

- At high risk for aspiration. 


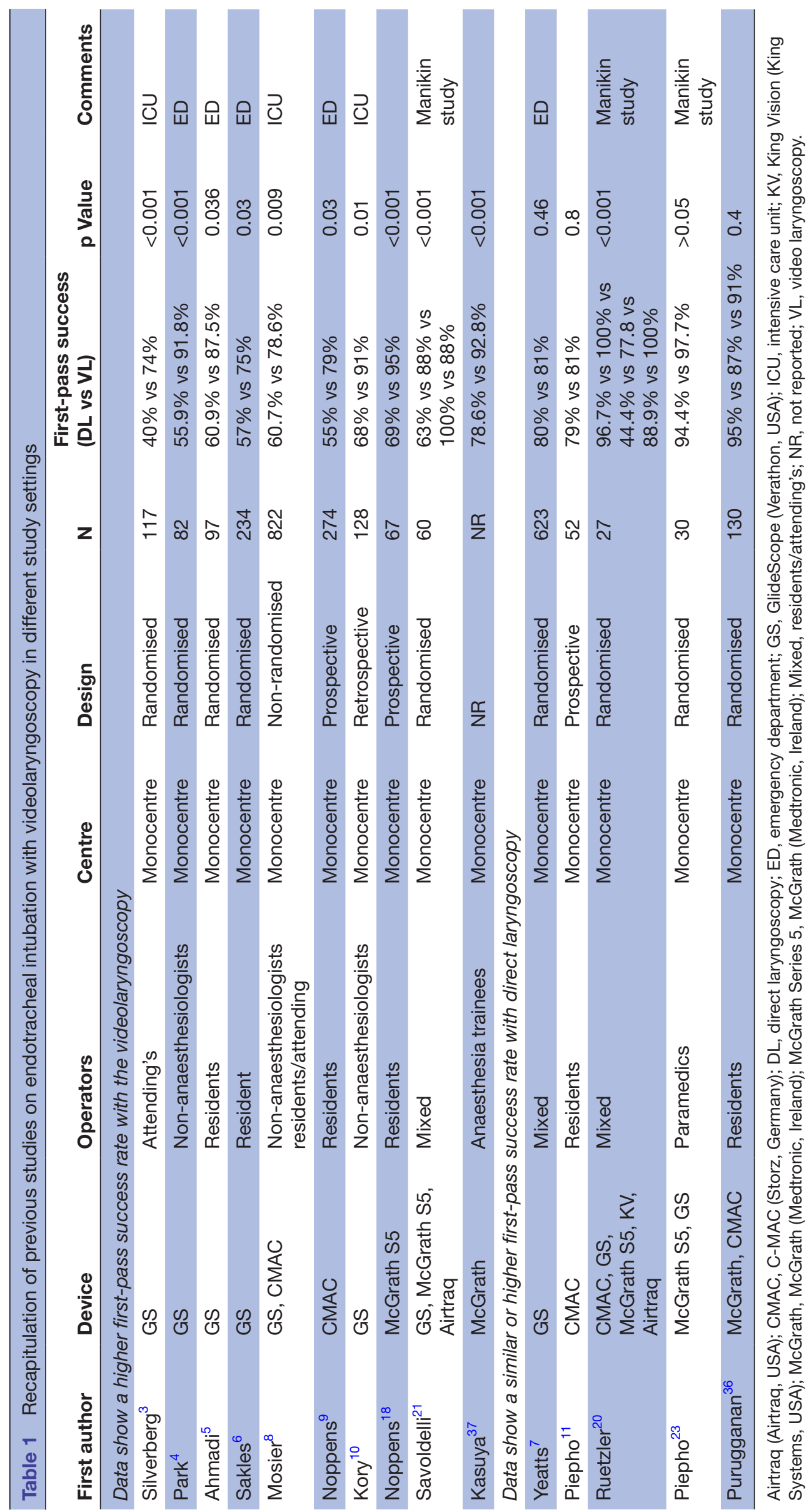




\section{Intervention}

\section{Concomitant treatments in both groups}

First, patients admitted requiring elective tracheal intubation are evaluated for predictors of anticipated difficult intubation (BMI, head extension, thyromental distance, Mallampati class, mouth opening and previous difficult airway (eg, C\&L $\geq$ III). The expertise of the participating anaesthesiologists ranges from 'beginner' (residents) to 'expert' (consultants). All anaesthetists received hands-on training and theoretical introduction to the use of the McGrath video laryngoscopy and DL. Tracheal intubation is performed in both groups following the protocol outlined below (figure 1).

1. All patients are monitored for ECG, oxygen saturation $\left(\mathrm{SO}_{2}\right)$ and arterial blood pressure (non-invasive or invasive as appropriate). In the McGrath group, a malleable stylet in a 'hockey-stick' shape is always used for tube placement.

Preoxygenation is achieved using the device chosen by the provider based on patient characteristics and clinical standard operating procedure $\left(\mathrm{EtO}_{2}>80 \%\right)$. In the study locations a Pallas/Primus (Dräger Lübeck, Germany) anaesthesia respiratory system is used:

- Tidal volume breathing with normal breaths for at least $3 \mathrm{~min}$ or with eight deep breaths over $60 \mathrm{~s}$ (8DB $60 \mathrm{~s})^{2829}$

- Anaesthesia ventilator in pressure support (PS) mode (PS eight mbar, Positive end-expiratory pressure 5 mbar and $\mathrm{FiO}_{2}$ 1.0). ${ }^{29} 30$

2. After sufficient preoxygenation, anaesthesia is induced with sufentanil $(0.2-0.5 \mu \mathrm{g} / \mathrm{kg})$ and propofol $(2-3 \mathrm{mg} / \mathrm{kg})$, and anaesthesia is maintained with either propofol infusion (TIVA) or volatile anaesthetics. After the patient is deeply anaesthetised, the neuromuscular transmission is monitored using acceleromyography of the adductor pollicis. The individual choice of neuromuscular blocking agent depends on the temporal duration of the surgery, necessary of perioperative neurological monitoring, absence of allergies and organ failures. The following agents and specific dosages are used:

- Mivacurium $(0.2 \mathrm{mg} / \mathrm{kg})$

- Atracurium $(0.5 \mathrm{mg} / \mathrm{kg})$

- Rocuronium $(0.3-0.6 \mathrm{mg} / \mathrm{kg})$

- Succinylcholine (1-2 mg/kg).

The train-of-four (TOF) is used for continuous quantitative monitoring of neuromuscular transmission. Complete muscle relaxation is confirmed in the absence of tactile and measured twitches in response to maximal TOF stimulation of the ulnar nerve at the adductor pollicis. The importance of obtaining adequate neuromuscular blockade is emphasised with study personnel.

3. The laryngoscopy attempt begins with a TOF count of $0 / 4$ and is performed using the device indicated by default randomisation:

- Macintosh laryngoscope (DL)

- McGrath MAC video laryngoscope (McGrath) - indirect laryngoscopy is initially performed. However, direct laryngoscopy can also be usedat the discretion of the anaesthetist.

The anaesthetist should achieve the best possible view of the laryngeal structures. External laryngeal manipulations (ELM) could be used to improve the view of the glottis to achieve a C\&L I or II. The size of the endotracheal tube and the size of the blade are dependent on the standard operating procedure of the hospital (blade size in both groups: \#3 for average patients and \#4 for very tall patients $(>190 \mathrm{~cm}$ height $)$; standard ETT size: 7.0 ID used for female patients and 7.5 ID for male patients). The method of visualisation of the glottis and size of the ETT/ blade is recorded in the case report form (CRF).

4. The laryngoscopy attempt is defined as successful if the tracheal tube is placed (until the black mark on the ET was threaded between the vocal cords) with a single blade insertion within $120 \mathrm{~s}$ and without manipulation of the laryngoscope by another provider. The 'time to intubation' is defined as the time measured from the opening of the patient's mouth until the ETT passed the vocal cords. An anaesthesia nurse measures the intubation time using the built-in timer on the anaesthesia respirator. We also analyse three time periods until final placement:

- 'Time to view': defined as the time from insertion of the device until visualisation of the glottis

- 'Time to intubation': defined as the time from insertion of the device until the ETT passed through the vocal cords

- 'Time to ventilation': defined as the time from insertion of the ETT until the time from the insertion of the device into the mouth until confirmation of the first wave of $\mathrm{CO}_{2}$ of the anaesthesia respirator.

An intubation attempt is defined as an introduction of the laryngoscope blade into the mouth and its removal regardless of whether an ETT was successfully inserted. If this first attempt fails, the provider makes a second laryngoscopy attempt with the same device. Mask ventilation is recommended between the attempts. A total of two laryngoscopy attempts are allowed. If DL fails, the clinician changes to a preferred technique (eg, McGrath, S-Guide, rigid stylet) and records the direct and/or screen view of the McGrath. If McGrath fails after two attempts, the clinician is advised to proceed with a preferred rescue technique (eg, CMAC D-Blade, SGA, iLMA, flexible fibreoptic, rigid stylet). The limitation of two intubation attempts and choice of an alternative technique is recommended by the study protocol and is in accordance with the clinical standard. ${ }^{31}$ If ELM techniques, such as BURP (specific pressure applied to the cricoid cartilage), are required during laryngoscopy, they are recorded in the CRF. In all cases, an additional individual who is not involved in patient care (either a postgraduate student or a study nurse) is present during induction of anaesthesia to record the study parameters. 
Figure 1: Study flow-chart

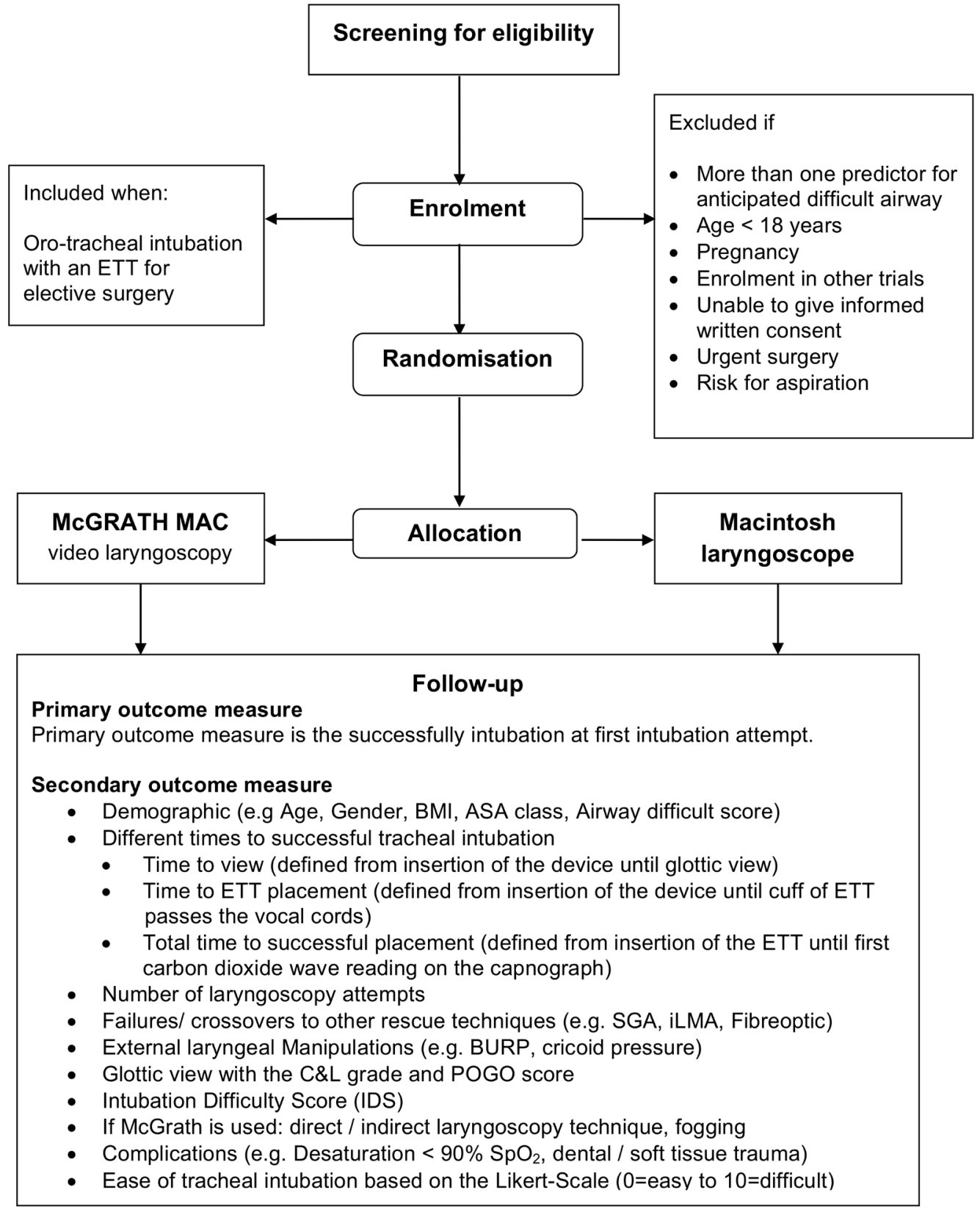

Figure 1 Study flow chart. ASA, American Society of Anesthesiologists; BMI, body mass index; BURP, backward, upward and rightward pressure; C\&L, Cormack \& Lehane; DL, direct laryngoscopy; ETT, endotracheal tube; POGO, percentage of glottic opening; SGA, supraglottic airway. 


\section{Outcome measures}

\section{Primary outcome measure}

The primary outcome measure is successful intubation within $120 \mathrm{~s}$ (time to ventilation) with the first-pass attempt.

\section{Secondary outcome measure}

- Laryngoscopy technique: whether direct or indirect glottic visualisation was used in the McGrath group is recorded

- Different times for successful tracheal intubation

- time to view (defined as the time from insertion of the device until glottic view)

- time to intubation (defined as the time from insertion of the device until the ET passed through the vocal cords)

- time to ventilation (defined as the time from insertion of the device until the first $\mathrm{CO}_{2}$ wave on the anaesthesia respirator)

- Number of laryngoscopy attempts

- Failures/crossovers to other rescue techniques (eg, SGA, iLMA, fibreoptic)

- ELM (eg, BURP, cricoid pressure)

- Glottic view with the C\&L and percentage of glottic opening score

- Intubation Difficulty Score (IDS) ${ }^{32}$

- If McGrath is used, occurrence of fogging is recorded

- Comparing the level of training with intubation success

- Complications (eg, desaturation $<90 \% \mathrm{SaO}_{2}$, dental or soft tissue trauma)

- Degree of ease or difficulty of tracheal intubation based on the Likert scale $(0=\text { easy to } 10=\text { difficult })^{33} 34$
Subgroup analysis

- Demographics

- patient (age, gender, BMI, ASA class)

- airway difficulty score (ADS) ${ }^{35}$

- provider analysis (clinical experience, education status, experience in direct and indirect laryngoscopy)

- Type of neuromuscular blocking agent

- TOF count when inserting the laryngoscope

- Type of surgery (eg, thyroidectomy, neck dissection).

\section{Participant timeline}

The schedule of enrolment and intervention is shown in figure 1, and the participant timeline is described in table 2 .

\section{Recruitment}

Patient inclusion started in 2016 in the Division of Ear, Nose and Throat surgery at University Medical Centre, Mainz, Germany, and inclusion of other divisions over the course of time is planned. The history and physical examinations of all patients scheduled for surgery are screened preoperatively for predictors of difficult airway. Patient recruitment is conducted by a study physician. Patients are included if they require orotracheal intubation with an ETT under general anaesthesia with neuromuscular blocking agents.

\section{Assignment of interventions \\ Allocation}

After eligibility is confirmed and written informed consent is obtained, enrolled participants are randomised 24 hours before the intervention. A web-based service (QuickCalcs,

Table 2 Participant timeline

\begin{tabular}{|c|c|c|c|c|c|c|}
\hline \multirow[b]{3}{*}{ Timepoint } & \multicolumn{5}{|c|}{ Study period } & \multirow{3}{*}{ Extubation } \\
\hline & Enrolment & Intervention & Intubation & & & \\
\hline & $D_{0}$ & $D_{1}$ & $\begin{array}{l}D_{1} \\
\text { (Time to } \\
\text { view) }\end{array}$ & $\begin{array}{l}D_{1} \\
\text { (Time to } \\
\text { intubation) }\end{array}$ & $\begin{array}{l}D_{1} \\
\text { (Time to } \\
\text { ventilation) }\end{array}$ & \\
\hline Eligibility assessment & $x$ & & & & & \\
\hline Informed consent & $x$ & & & & & \\
\hline Randomisation & $x$ & & & & & \\
\hline $\begin{array}{l}\text { Demographic data and } \\
\text { physical examination }\end{array}$ & $x$ & & & & & \\
\hline Preoxygenation & & $x$ & & & & \\
\hline Induction of anaesthesia & & $x$ & & & & \\
\hline TOF measurement & & $x$ & & & & \\
\hline Time measurement & & & $x$ & $\mathrm{X}$ & $x$ & \\
\hline Glottic view & & & $x$ & & & \\
\hline Intubation success & & & & & $x$ & \\
\hline Complications & & & $x$ & $\mathrm{x}$ & $x$ & $x$ \\
\hline
\end{tabular}

$D$, day; $D_{0}$, day of enrolment/allocation; $D_{1}$, day of surgery; $D_{2}$, intensive care unit stay; TOF, train-of-four. 
GraphPad Software, La Jolla, California, USA) is used for allocating patients to either DL or McGrath.

\section{Sequence generation}

The randomisation sequence was generated by a study nurse in the clinical research unit who is not involved in patient recruitment. The software used to collect the data in the CRF automatically allocated the patients, thereby ensuring concealment and anonymity.

\section{Blinding}

Blinding to the type of laryngoscopy is only possible for the patient. The performing anaesthesiologist is informed of treatment group prior to induction of anaesthesia.

\section{Data collection, management and analysis \\ Data collection and management}

The study data are recorded on a specific paper-based CRF. Prior to measurement, the data from each patient are collected by study personnel. All outcome measurements are recorded during and after the evaluation on the CRF. Any protocol deviations are recorded either on the CRF or in the medical records; a clinical research assistant ensures that all protocol deviations and adverse events are recorded in the database. If adverse events are observed, the ethics committee will be informed in writing.

Every allocated subject will be coded with a specific patient number. After measurement is completed, the study data will be entered into a premade computer-based table (Microsoft Excel, V.14.0, Microsoft, Redmond, Washington, USA). The completed CRF will be secured in the clinical research unit for the next 15 years.

\section{Access to data}

Data safety, data quality and statistical analysis will be managed by the two principal investigators, who are responsible for notifying any issues that may arise during the whole prospective study. Data are collected and stored according to good clinical practice (GCP) guidelines and are available to all participating study sites. Any issue occurring during the clinical trial will be reported to the principal investigators.

\section{Statistics}

For statistical analysis, GraphPad Prism (V.6.0 for MAC; GraphPad Software) will be used. Data are expressed as the median (IQR) for non-Gaussian variables. The statistical analysis will conform to the CONSORT statement for non-pharmacological interventions.

\section{Description of the patient groups at baseline}

The baseline features of the patients will be described using absolute numbers (n) and percentages for categorical variables and the minimum, maximum, mean, SD and quartiles for quantitative variables. We will use the Pearson correlation coefficient to compare patient specifics (eg, BMI, ADS score) between the groups at the baseline.
Analysis of the primary outcome

A $\chi^{2}$ test will be used to compare the success rate between the two groups. Multiple regression analysis of subgroup factors will allow the determination of important factors affecting successful first-time intubation comparing DL with McGrath. Relationships between the experience of the provider and the first-attempt intubation rate or time to intubation will be analysed as paired samples with Bonferroni correction for multiple comparisons. The differences will be considered statistically significant if the $\mathrm{p}$ value is less than 0.05 .

\section{Analysis of the secondary outcomes}

Comparison of the view of the glottis, overall intubation time and the Likert scale score will be analysed by the Wilcoxon's rank-sum test. Comparing the different levels of training with the intubation success will be done with the Spearman's rank correlation coefficient.

\section{Subgroup analysis}

We will perform a separate analysis of the specific type of surgery (eg, thyroidectomy, neck dissection), influence of neuromuscular agents and/or patients with difficult intubation, defined as more than two attempts or IDS score $>5$.

\section{Sample size}

A sample size calculation was based on achieving successful tracheal intubation on the first attempt within $120 \mathrm{~s}$ (time to ventilation) compared with more than one attempt. We determined the power of the study by assuming a first-pass success rate of $85 \%(\mathrm{DL})^{13} 14$ and 90\% (McGrath). ${ }^{36}{ }^{37}$ On the basis of the current first-pass success rate, we hypothesised that an increase of $5 \%$ by skilled laryngoscopists in the McGrath group compared with the DL group would be a relevant improvement in airway management. We determined that the inclusion of 1000 patients per group would show relevant differences. With 1000 patients, an increase of 85\%-90\% (DL) and 90\%-95\% (McGrath) in the first-pass success rate can be observed with a power of $96 \%$ at the $1.67 \%$ significance level.

\section{Monitoring}

Data monitoring

Prior to the start of patient enrolment, the study physicians and the clinical research assistants were involved in the study protocol and data collection in CRFs. All documents required for the study (eg, informed consent, CRF baseline and perioperative) are available in the operating room, where the study measurement begins. The CRF is prepared and managed by the investigator. Because this is an investigator-initiated trial, the principal investigator meets with clinical research assistants to discuss any problems in data collection and protocol compliance and to evaluate study progress. This study is proposed, managed and will be analysed in accordance with the ICH Guideline for GCP E6 (R2) and following the requirements of 
German law. All persons (eg, investigator, study assistants) are obliged to follow these rules.

\section{Harms}

The study may be temporarily stopped for an individual patient, at the discretion of the attending physician, in case of major serious adverse events suspected to be associated with the type of laryngoscope used. An adverse event or suspected adverse reaction is considered 'serious' if, in the view of either the investigator or sponsor, it results in any of the following outcomes: death, a life-threatening adverse event, inpatient hospitalisation or prolongation of existing hospitalisation, and a persistent or significant incapacity or substantial disruption of the ability to conduct normal life functions.

Reporting of severe adverse events (SAE) will be per local research ethics committee (REC) standard operating procedures. SAEs will include the following when occurring as a result of airway manipulation (eg, cardiac arrest, acute circulatory failure, death, vocal cord injury, oesophageal rupture). The principal investigator informs the REC about the SAE. No specific reporting procedure for unexpected serious adverse events is planned.

\section{Auditing}

The Clinical Research Unit of the Department of Anaesthesiology, University Medical Centre Mainz reviews the screening form and clinical data at regular intervals.

\section{ETHICS AND DISSEMINATION \\ Research ethics approval}

This study is conducted in adherence with the current version of the Declaration of Helsinki and GCP guidelines. The initial research project was approved by the ethics committee (Medical Association of the State of Rhineland Palatine, Germany) in October 2015 (Registration No: 837.296.15 (10064); NCT 02611986). It was also approved by the Medical Association of Westphalia, Lippe, Germany, in March 2016 (Registration No: 2016110-b-S).

\section{Consent or assent}

Prior to the trial, patients must consent orally and in writing after the possible consequences of the clinical study are explained in an understandable way. All documents must be written in German and comprehensible. According to German law, only a physician can have the conversation with the participant. The patient receives a copy of the signed patient information and informed consent. A patient may withdraw from the study at any time if he is unwilling to continue in the trial. In this case, the data from a patient who requests full withdrawal will not be considered in the data analysis.

\section{Confidentiality}

All original documents will be kept in the clinical research unit for the next 15 years.
The study data will be handled as requested by the German Federal Data Protection Act, which implements the Directive 95/46/EC on data protection (Data Protection Directive). All original records will be kept on file at the trial sites or coordinating data managing centre for 15 years. The cleaned electronic trial database file will be anonymised and kept on file for 15 years.

\section{Declaration of interests}

Neither the participating medical centers nor any of its employees received any compensation for this work. No funding or competing interests are declared. None of the authors have financial interests or received honoraria or paid expert testimony. None of the authors have any personal relationships with people or organisations that could inappropriately influence (bias) this work. Covidien, which produces the McGrath video laryngoscope, had no role in the study design and will have no role in its conduct, data collection, analysis or interpretation, or the decision to submit the results for publication. The findings of this study will be presented at conferences and disseminated through publication in a peer-reviewed journal.

\section{DISCUSSION}

Several studies have suggested that video laryngoscopy and DL using a Macintosh blade had similar intubation success rates. ${ }^{7112023}$ The weaknesses of the existing research include the study setting (eg, manikin-based study or measurement in intensive care unit) $)^{9} 101920$ and study design (eg, inadequate sample size or variables in anaesthesia induction). ${ }^{3523}$ Furthermore, the clinical experience of the user was not usually taken into account. ${ }^{4} 6102325$ To our knowledge, the EMMA study is the only clinical, multicentre, randomised study with 2000 patients comparing video laryngoscopy and DL for the first-attempt tracheal intubation. This trial has an openlabel design; blinding of the operator or the patient is not feasible. However, the primary outcome measure is the presence of the inflection on the expired capnography curve to ensure that the ET is in the tracheal position. The main outcome of other studies was the duration of the intubation attempt. For detailed information about the overall intubation time, we divide the overall time into three time periods, from insertion of the laryngoscope until the first ventilation. The visualisation of the glottis is another preferred outcome parameter in several airway studies, but a good view of the glottis cannot be associated with successful or faster tracheal intubation. ${ }^{20}{ }^{21}$ Furthermore, the number of attempts constitutes a relevant factor for increased airway complications (eg, risk of aspiration, tissue/mucosal damage) and desaturation during the intubation process. ${ }^{14-17}$

In conclusion, if our main hypothesis is confirmed, video laryngoscopy might become the reference standard in the operating room. The expected benefits of this practice include improved instruction of airway management 
and influence of neuromuscular agents for the intubation procedure, as well as improved patient safety in terms of decreased airway complications (eg, hypoxaemia, aspiration).

Acknowledgements The authors wish to thank all anesthesiologists from the study sites for their great effort and support of this trial.

Contributors MK and RRN designed the trial and prepared the manuscript. TP, IT and CA participated in designing the EMMA trial. IS wrote the statistical analysis plan and estimated the sample size. None of the authors have financial interests or received honoraria or paid expert testimony. None of the authors have any personal relationships with people or organisations that could inappropriately influence (bias) this work. The authors alone are responsible for the content and writing of the paper.

Competing interests None declared.

Patient consent Obtained.

Ethics approval Medical Association of the State of Rhineland Palatinate, Germany, Registration Nr.: 837.296.15 (10064); NCT 02611986.

Provenance and peer review Not commissioned; externally peer reviewed.

Open Access This is an Open Access article distributed in accordance with the Creative Commons Attribution Non Commercial (CC BY-NC 4.0) license, which permits others to distribute, remix, adapt, build upon this work non-commercially, and license their derivative works on different terms, provided the original work is properly cited and the use is non-commercial. See: http://creativecommons.org/ licenses/by-nc/4.0/

(C) Article author(s) (or their employer(s) unless otherwise stated in the text of the article) 2017. All rights reserved. No commercial use is permitted unless otherwise expressly granted.

\section{REFERENCES}

1. Mulcaster JT, Mills J, Hung OR, et al. Laryngoscopic intubation: learning and performance. Anesthesiology 2003;98:23-7.

2. Konrad C, Schüpfer $G$, Wietlisbach $M$, et al. Learning manual skills in anesthesiology: is there a recommended number of cases for anesthetic procedures? Anesth Analg 1998;86:635-9.

3. Silverberg MJ, Li N, Acquah SO, et al. Comparison of video laryngoscopy versus direct laryngoscopy during urgent endotracheal intubation: a randomized controlled trial. Crit Care Med 2015;43:636-41.

4. Park SO, Kim JW, Na JH, et al. Video laryngoscopy improves the first-attempt success in endotracheal intubation during cardiopulmonary resuscitation among novice physicians. Resuscitation 2015;89:188-94.

5. Ahmadi K, Ebrahimi M, Hashemian AM, et al. GlideScope Video Laryngoscope for Difficult Intubation in Emergency Patients: a Quasi-Randomized Controlled Trial. Acta Med Iran 2015;53:738-42.

6. Sakles JC, Mosier JM, Chiu S, et al. Tracheal intubation in the emergency department: a comparison of GlideScope $₫$ video laryngoscopy to direct laryngoscopy in 822 intubations. J Emerg Med 2012;42:400-5.

7. Yeatts DJ, Dutton RP, Hu PF, et al. Effect of video laryngoscopy on trauma patient survival: a randomized controlled trial. J Trauma Acute Care Surg 2013;75:212-9.

8. Mosier JM, Whitmore SP, Bloom JW, et al. Video laryngoscopy improves intubation success and reduces esophageal intubations compared to direct laryngoscopy in the medical intensive care unit. Crit Care 2013;17:R237.

9. Noppens RR, Geimer S, Eisel N, et al. Endotracheal intubation using the C-MAC $®$ video laryngoscope or the Macintosh laryngoscope: a prospective, comparative study in the ICU. Crit Care 2012;16:R103.

10. Kory P, Guevarra K, Mathew JP, et al. The impact of video laryngoscopy use during urgent endotracheal intubation in the critically ill. Anesth Analg 2013;117:144-9.

11. Piepho T, Fortmueller K, Heid FM, et al. Performance of the C-MAC video laryngoscope in patients after a limited glottic view using Macintosh laryngoscopy. Anaesthesia 2011;66:1101-5.

12. Michailidou M, O'Keeffe T, Mosier JM, et al. A comparison of video laryngoscopy to direct laryngoscopy for the emergency intubation of trauma patients. World J Surg 2015;39:782-8.
13. Vassiliadis J, Tzannes A, Hitos K, et al. Comparison of the C-MAC video laryngoscope with direct Macintosh laryngoscopy in the emergency department. Emerg Med Australas 2015;27:119-25.

14. Platts-Mills TF, Campagne $D$, Chinnock B, et al. A comparison of GlideScope video laryngoscopy versus direct laryngoscopy intubation in the emergency department. Acad Emerg Med 2009;16:866-71.

15. Mort TC. Emergency tracheal intubation: complications associated with repeated laryngoscopic attempts. Anesth Analg 2004;99:607-13.

16. Hasegawa K, Shigemitsu K, Hagiwara Y, et al. Association between repeated intubation attempts and adverse events in emergency departments: an analysis of a multicenter prospective observational study. Ann Emerg Med 2012;60:749-54.

17. Sakles JC, Javedani PP, Chase E, et al. The use of a video laryngoscope by emergency medicine residents is associated with a reduction in esophageal intubations in the emergency department. Acad Emerg Med 2015;22:700-7.

18. Noppens RR, Möbus S, Heid F, et al. Evaluation of the McGrath Series 5 videolaryngoscope after failed direct laryngoscopy. Anaesthesia 2010;65:716-20.

19. Kaplan A, Göksu E, Yıldız G, et al. Comparison of the C-MAC Videolaryngoscope and rigid fiberscope with direct laryngoscopy in Easy and Difficult Airway scenarios: a Manikin Study. J Emerg Med 2016;50:e107-e114.

20. Ruetzler K, Imach S, Weiss M, et al. [Comparison of five video laryngoscopes and conventional direct laryngoscopy : investigations on simple and simulated difficult airways on the intubation trainer]. Anaesthesist 2015;64:513-9.

21. Savoldelli GL, Schiffer E, Abegg C, et al. Comparison of the Glidescope, the McGrath, the Airtraq and the Macintosh laryngoscopes in simulated difficult airways*. Anaesthesia 2008;63:1358-64.

22. Aziz MF, Abrons RO, Cattano D, et al. First-Attempt Intubation success of Video Laryngoscopy in patients with Anticipated Difficult Direct Laryngoscopy: a Multicenter Randomized Controlled Trial comparing the C-MAC D-Blade Versus the GlideScope in a Mixed Provider and Diverse Patient Population. Anesth Analg 2016;122:740-50.

23. Piepho T, Weinert K, Heid FM, et al. Comparison of the McGrath ${ }$ Series 5 and GlideScope $®$ Ranger with the Macintosh laryngoscope by paramedics. Scand J Trauma Resusc Emerg Med 2011;19:4.

24. Savoldelli GL, Schiffer E, Abegg C, et al. Learning curves of the Glidescope, the McGrath and the Airtraq laryngoscopes: a manikin study. Eur J Anaesthesiol 2009;26:554-8.

25. Wallace CD, Foulds LT, McLeod GA, et al. A comparison of the ease of tracheal intubation using a McGrath MAC(®) laryngoscope and a standard Macintosh laryngoscope. Anaesthesia 2015;70:1281-5

26. Bailly A, Lascarrou JB, Le Thuaut A, et al. McGRATH MAC videolaryngoscope versus Macintosh laryngoscope for orotracheal intubation in intensive care patients: the randomised multicentre MACMAN trial study protocol. BMJ Open 2015;5:e009855.

27. Chan AW, Tetzlaff JM, Gøtzsche PC, et al. SPIRIT 2013 explanation and elaboration: guidance for protocols of clinical trials. BMJ 2013;346:e7586.

28. McCrory JW, Matthews JN. Comparison of four methods of preoxygenation. Br J Anaesth 1990;64:571-6.

29. Tanoubi I, Drolet P, Donati F. Optimizing preoxygenation in adults. Can J Anaesth 2009;56:449-66.

30. Murphy C, Wong DT. Airway management and oxygenation in obese patients. Can J Anaesth 2013;60:929-45.

31. Piepho T, Cavus E, Noppens R, et al. S1 guidelines on airway management : Guideline of the German Society of Anesthesiology and Intensive Care Medicine. Anaesthesist 2015;64:27-40.

32. Adnet F, Borron SW, Racine SX, et al. The intubation difficulty scale (IDS): proposal and evaluation of a new score characterizing the complexity of endotracheal intubation. Anesthesiology 1997:87:1290-7.

33. Weiss M, Schwarz U, Gerber AC. Difficult airway management: comparison of the Bullard laryngoscope with the video-optical intubation stylet. Can J Anaesth 2000;47:280-4.

34. Makwana HD, Suthar NN, Gajjar MP, et al. Developing competency in interns for endotracheal intubation: an educational article. Int J Appl Basic Med Res 2016;6:201-4.

35. Janssens M, Lamy M. Airway Difficulty Score (ADS): a new score to predict difficulty in airway management. Eur $J$ Anaesthesiol 2000;17:35

36. Purugganan RV, Jackson TA, Heir JS, et al. Video laryngoscopy versus direct laryngoscopy for double-lumen endotracheal tube intubation: a retrospective analysis. J Cardiothorac Vasc Anesth 2012;26:845-8. 
37. Kasuya Y, Takahashi E, Nagai M, et al. [Comparison of Tracheal Intubation Performance between Macintosh Direct Laryngoscope and McGRATH $®$ MAC Video Laryngoscope among Anesthesia Trainees]. Masui 2015;64:1291-6. 